- Intravenous sedation with Midazolam is well established but is not suitable for all clinical situations.

- Patient-maintained sedation with propofol may offer an alternative to midazolam.

- The system is safe and reproducible.

- Patient and surgeon satisfaction are high.

- Recovery is fast and uneventful.

\title{
Patient-maintained sedation for oral surgery using a target-controlled infusion of propofol - a pilot study
}

\author{
J. A. Leitch, ${ }^{1}$ N. Sutcliffe ${ }^{2}$ and G. N. C. Kenny ${ }^{3}$
}

Objective To assess the safety and efficacy of a new patient-maintained propofol system for conscious sedation in dentistry.

Design Prospective clinical trial.

Setting Department of Sedation, Glasgow Dental Hospital and School, 2001.

Subjects and methods Patients scheduled for oral surgery with conscious sedation. Exclusions included ASA IV - V, inability to use the handset, opioid use and severe respiratory disease.

Interventions Patients were given intravenous propofol to a level of $1.0 \mu \mathrm{g} / \mathrm{ml}$ (reducing from $1.5 \mu \mathrm{g} / \mathrm{ml}$ ) using a target controlled infusion system, they then controlled their sedation level by double-clicking a handset which on each activation increased the propofol concentration by $0.2 \mu \mathrm{g} / \mathrm{ml}$.

Main outcome measures Oxygen saturation, patient satisfaction, and surgeon satisfaction.

Results Twenty patients were recruited, 16 female and four male. Nineteen patients completed sedation and treatment successfully. Mean lowest oxygen saturation was 94\%. No patients were over-sedated. All patients successfully used the system to maintain a level of sedation adequate for their comfort. Patient and surgeon satisfaction were consistently high.

Conclusions Initial experience with this novel system has confirmed safety, patient satisfaction and surgeon satisfaction.

Patients receiving intravenous conscious sedation for dentistry within the UK in general practice, community practice and the hospital service tend to receive incremental doses of intravenous midazolam administered by an operator-sedationist. Although there have been no major adverse events in dentistry the safety of such a regime has been questioned in other specialities with reports of up to $13 \%$ of patients desaturating to below $80 \%$ in the recovery area following sedation for endoscopy. ${ }^{1}$ Mortality has been reported in $0.05 \%$ of patients with approximately $60 \%$ of this

${ }^{1}$ Clinical Lecturer, Glasgow Dental Hospital and School, ${ }^{2}$ Consultant Anaesthetist, $\mathrm{HCl}$ Hospital ${ }^{3}$ Professor of Anaesthesia, University Department of Anaesthesia, Royal Infirmary, Glasgow

${ }^{*}$ Correspondence to: Mr Jason Leitch, Clinical Lecturer, Glasgow Dental Hospital and School, 378 Sauchiehall Street, Glasgow G2 3JZ

E-mail:leitch@bigfoot.com

\section{Refereed paper}

Received 08.03.02; Accepted 23.09.02

( ) British Dental Journal 2003; 194: 43-35 being due to hypoxaemia. ${ }^{1-4}$ No such serious outcomes have been reported in dentistry.

In anaesthesia, target-controlled infusion (TCI) of propofol is gaining wide popularity. The system uses a pharmacokinetic model to achieve and maintain a selected target blood propofol concentration. The system has been further developed to incorporate a handset so that patients can titrate their own blood propofol concentrations to a sedation level appropriate to themselves. This combines the benefits of TCI with the well-established safety of a patient-controlled feedback loop and has been termed patientmaintained sedation (PMS).

Low dose propofol has been shown to have satisfactory anxiolytic properties when used as a sedative. ${ }^{5}$ It has been evaluated in a variety of intermittent bolus and infusion regimes. ${ }^{6-8}$

Patient-controlled sedation with propofol TCI has been shown to be safe and effective in reducing pre-operative anxiety levels when used to provide intra-operative sedation for patients undergoing surgery, with no patients becoming oversedated, premedication for day case patients and most recently in patients undergoing endoscopic examination. ${ }^{9-11}$

Propofol has several pharmacokinetic parameters that potentially make it a superior sedative agent to midazolam; more rapid onset and offset being two. However its routine use has been restricted to anaesthetists, as in high doses, general anaesthesia is produced with possible loss of the patient's patent airway.

Conscious sedation for minor oral surgery procedures is becoming increasingly popular as general anaesthesia becomes less so. In combination with local anaesthesia it potentially forms a pain-free and anxiety-free technique for both the patient and the surgical team. Any new drug delivery system must be carefully assessed for safety and efficacy in this specific environment.

This study assesses the first use of this patient-maintained sedation system using TCI propofol for oral surgery in 20 patients as a sedative technique for these procedures.

\section{MATERIALS AND METHODS}

The project was approved by the Hospital Ethics Committee. Twenty patients who were referred for oral surgery with conscious sedation were asked to participate in a study using patient-maintained propofol as the sedative technique. Recruitment was not selective and therefore included a range of anxiety levels from those phobic of dentistry to those anxious about surgical procedures. Written 
consent was obtained after a full explanation of the sedative technique and the surgery involved. Any patient considered fit for sedation was recruited. All patients were required to bring an escort and standard post-operative sedation instructions were given. These included no driving for twelve hours and resting at home for the rest of the day. Patients were not starved prior to treatment but were advised to have a light meal two hours before their appointment. Exclusion criteria included inability to use the handset, opioid use and severe respiratory disease.

Venous access was obtained and baseline observations were obtained of blood pressure, oxygen saturation and heart rate. Propofol TCI was delivered via a Graseby 3400 infusion pump controlled by a microprocessor system programmed with the pharmacokinetic data describing the distribution and elimination of propofol. ${ }^{10,12}$ The system displays the calculated effect-site, or the theoretical brain concentration, in addition to the target blood concentration of propofol, based on the patient's age and weight which are entered into the microprocessor. Connection of a handset to the microprocessor allows the patient to increase their target blood concentration of propofol (Figure 1).

Propofol (1\% with lidocaine $20 \mathrm{mg}$ per $50 \mathrm{ml}$ to decrease pain on injection) was started at an initial target blood concentration of $1.5 \mathrm{\mu g} / \mathrm{ml}$. When the microprocessor estimated the effect-site concentration to be $1.0 \mu \mathrm{g}$ the target was reduced to $1.0 \mu \mathrm{g}$ and the patient was instructed to 'double-press' the button as often as necessary. They were advised to sedate themselves to an appropriate level at which they would allow local anaesthesia to be administered.

Successful activation of the handset by double pressing the button within one second would increase the target blood concentration of propofol by $0.2 \mu \mathrm{g} / \mathrm{ml}$. There was a lock-out period of two minutes during which no further target increases were possible. The maximum permissible target blood concentration of propofol was $3.0 \mu \mathrm{g} / \mathrm{ml}$. If no successful demands were detected in six minutes the microprocessor would automatically reduce the target blood concentration by $0.2 \mu \mathrm{g} / \mathrm{ml}$.

Once the patient considered themselves ready, they received a standard dental local anaesthetic (2\% lidocaine with 1:80,000 adrenaline) and the surgery was carried out. They were advised to press the button at any stage if they wanted to be more sedated.

The patients were monitored throughout with pulse oximetry and measurements of heart rate and arterial oxygen saturation were recorded every five minutes. Blood pressure readings were recorded pre-operatively and prior to discharge. Any emergency interventions were noted. Patients were taken to a dedicated recovery area after their surgery was complete where they were monitored throughout with pulse oximetry by a trained dental nurse.

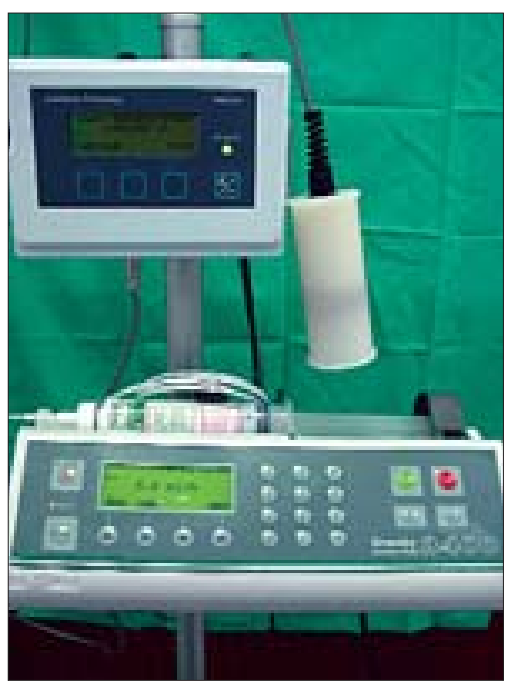

Figure 1 Patient maintained sedation equipment including handset.
Once the patient was judged fit for discharge using a modified Romberg's test and by demonstrating a steady, unaided walk across the recovery room they completed a questionnaire designed to assess patient recall and satisfaction with the technique. The surgeon also completed a questionnaire regarding ease of undertaking the procedure and patient cooperation.

A note was made of start time, the time the patient-controlled button was enabled, surgery start time, surgery finish time and discharge time. The lowest recorded arterial oxygen saturation was also noted.

\section{RESULTS}

Of the 20 patients, 19 completed their treatment satisfactorily and maintained a level of sedation which was both adequate for their anxiety control and for completion of the procedure. There were 16 female and four male patients. The mean age was 31 years with a range from 12 to 58. Fourteen patients were ASA I, five were ASA II and one was ASA III.

One of the twenty patients was a known epileptic and on completion of the surgery suffered three progressively shorter grand mal seizures with increasing time between each. She recovered spontaneously on each occasion and no measures other than monitoring and reassurance were required. She was transferred by ambulance to the local accident and emergency department where she was supervised for two hours. She suffered no further seizures and was discharged home without further problems. She could not be considered to have completed the study since it was impossible to complete the post-operative questionnaire.

All surgical procedures were carried out by the same surgeon and a variety of surgical procedures were performed (Table 1).

\begin{tabular}{lc}
\multicolumn{2}{l}{ Table 1 Surgical procedures performed } \\
\hline Surgical Procedure & Number \\
\hline Third molar extraction & 12 \\
Multiple extractions & 4 \\
Impacted canine removal & 2 \\
Apicectomy & 1
\end{tabular}

The mean procedure time was 15.4 minutes (4-30 mins). The mean time to local anaesthetic was 8.3 minutes (5-16 mins), to discharge was 47.4 mins (35-80 mins) and from end of procedure to discharge was 22.2 minutes (10-45 mins).

There were no cases of haemodynamic instability and the mean lowest oxygen saturation was 94\%. One patient had a transient saturation of $87 \%$ which was corrected easily with supplemental oxygen at $21 / \mathrm{min}$ via nasal cannulae. This patient was the only ASA III patient in the study. The lowest arterial oxygen saturation for each patient is shown in Figure 2. There were no significant changes in heart rate or blood pressure.

All twenty patients used the system satisfactorily to maintain a level of sedation which was adequate for their comfort and the completion of the surgical procedure. The recorded effect-site concentrations of propofol did not vary much immediately before the injection of local anaesthetic but showed a three-fold variation by the end of the surgical procedure. (Table 2).

\begin{tabular}{|c|c|c|}
\hline Time & Median & Range \\
\hline Immediately before LA & 1.1 & $1-1.5$ \\
\hline At end of surgical procedure & 2 & $0.9-2.8$ \\
\hline
\end{tabular}

Of the nineteen patients who completed a post-operative questionnaire all were happy with the sedation they received and would have the same system again. No patients reported nausea at any stage. Four experienced pain on injection of the propofol and six reported some pain during the procedure. Using a four point 




Figure 2 Lowest arterial oxygen saturation

scale (no recall, not unpleasant, unpleasant, horrible) 18 patients found the whole procedure 'not unpleasant' and only one patient described it as 'unpleasant'. All patients recalled arriving in the dental surgery and the intravenous cannulation, 18 recalled injection of the local anaesthesia and seventeen reported remembering aspects of the surgical procedure. The patient who could not recall the local anaesthetic could recall the surgery.

Using a four point scale (easy, mild difficulty, difficult, very difficult) the surgeon rated three (16\%) as 'easy', ten (53\%) as 'mild difficulty' and six (32\%) as 'difficult'. Using another four point scale (very good, good, average, minimal) the surgeon rated patient co-operation as 'very good' in sixteen cases (84\%), 'good' in two cases (10\%) and 'minimal' in one case (5\%). The case rated as 'minimal' was a twelve-year-old for removal of four first molars.

A number of patients spontaneously volunteered when asked if they had any further comments that they liked the control they had over their sedation.

\section{DISCUSSION}

Initial evaluation of this patient-maintained sedation system for oral surgery procedures has provided useful practical data. The system was used successfully and treatment was completed in all 20 patients. The median effect-site blood concentration required for adequate sedation was lower than in previous studies. ${ }^{9-11}$ No patient reached the maximum level of $3.0 \mu \mathrm{g} / \mathrm{ml}$. Previous studies using endoscopy patients have reported levels of up to $4.5 \mu \mathrm{g} / \mathrm{ml} .{ }^{9}$ This observation is almost certainly due to the good local anaesthetic blocks achievable for oral surgery procedures.

Sedation in dentistry tends to be provided with incremental doses of benzodiazepines. This technique is associated with a high degree of amnesia. The results from the present study suggest that a high proportion of patients treated with patient-maintained propofol sedation at these levels recall aspects of their procedure and more importantly they report it as not unpleasant. This may be a distinct advantage in trying to educate patients about the painless nature of modern dentistry. Previous reports, principally of endoscopy, have shown higher levels of amnesia and it has been suggested that this is dose related. Willingness of all patients in the present study to have a repeat procedure with the PMS technique would suggest that the recall of the patients in this study is a positive outcome. Although oral surgery tends to be a one-off procedure more general dentistry can often require repeat visits. The willingness to repeat the experience is a distinct advantage.

The system was found to be safe in that no patient suffered serious desaturation. The only patient with a saturation below $90 \%$ was promptly and easily treated with supplemental oxygen. In previous studies all patients have received supplemental oxygen, ${ }^{9-11}$ but our data would suggest this is unnecessary in our group of patients. No patients were oversedated and all maintained verbal contact throughout the procedure.

The one patient who did not complete the study did however complete her surgery and sedation. All the difficulties were postoperatively. Caution with propofol in epilepsy is well document$\mathrm{ed}^{13}$ and our findings would suggest this system needs significant care in epileptic patients.

The surgeon rated cooperation as 'very good' in $84 \%$ of cases which is very encouraging. The only case in which cooperation was a problem was the only patient under 16 years old in the present study. This would fit with reports and should lead to caution in using the system in those under 16.

It is too early to recommend this system for wide use but initial experience has confirmed both surgeon and patient satisfaction were high. These results are encouraging and in combination with the increasing literature on the subject, the next stage is a prospective, randomised controlled trial of this new system against the more established technique of administering incremental doses of midazolam. The end-point is a safe and effective operator-sedationist system.

1. Quine M A, Bell G D, McCloy R F, Charlton J E, Devlin H B, Hopkins A. Prospective audit of upper gastrointestinal endoscopy in two regions of England: safety, staffing and sedation methods. Gut 1995; 95: 462-467.

2. Bell G D. Review article: premedication and intravenous sedation for upper gastrointestinal endoscopy. Ailment Pharmacol Ther 1990; 4: 103-122.

3. Murray A W, Morran C G, Kenny G N, Macfarlane P, Anderson J R. Examination of cardiorespiratory changes during upper gastrointestinal endoscopy. Comparison of monitoring of arterial oxygen saturation, arterial pressure and the electrocardiogram. Anaesthesia 1991; 46: 181-184.

4. Charlton J E. Monitoring and supplemental oxygen during endoscopy. Br Med J 1995; 310: 886-887

5. Smith I, Monk T G, White P F, Ding Y. Propofol infusion during regional anaesthesia: sedative, amnestic and anxiolytic properties. Anesth Analg 1994; 79: 313-319.

6. Keefe E B. Sedation and Analgesia for Endoscopy. Gastroenterology 1995; 108: 932 934

7. Church J A, Stanton P D, Kenny G N C, Anderson J R. Propofol for sedation during endoscopy: assessment of a computer-controlled infusion system. Gastrointest Endosc 1991: 37: 175-179

8. Roseveare C, Seavell C, Patel P, Criswell J, Shepherd H. Patient-controlled sedation with propofol and alfentanil during colonoscopy: a pilot study. Endosc 1998; 30 : 482-483.

9. Irwin M G, Thompson N, Kenny G N C. Patient-maintained propofol sedation. Anaesthesia 1997; 52: 525-530.

10. Murdoch J A C, Kenny G N C. Patient-maintained propofol sedation as premedication in day-case surgery: assessment of a target-controlled system. Br J Anaesth 1999; 82 429-431.

11. Gillham M J, Hutchinson R C Cater R, Kenny, G N. Patient-controlled sedation for ERCP using a target-controlled infusion of propofol: a pilot study. Gastrointestinal Endosc 2001; 54: 14-17.

12. White $M$, Kenny G N C. Intravenous propofol anaesthesia using a computerised infusion system. Anaesthesia 1990; 45: 204-209.

13. Sneyd J R. Propofol and epilepsy. Br J Anaesth 1999; 82: 168-169. 